\title{
Crop Growth Observers Required to Reach Full Automation of a Greenhouse
}

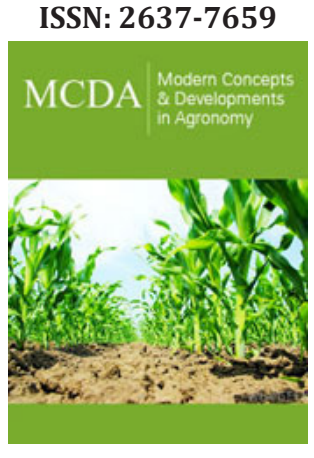

*Corresponding author: Divas Karimanzira, Fraunhofer IOSB, Am Vogelherd 90, 98693 Ilmenau, Germany

Submission: 鮆 April 28, 2021

Published: 想 May 04, 2021

Volume 8 - Issue 4

How to cite this article: Divas Karimanzira, Thomas Rauschenbach. Crop Growth Observers Required to Reach Full Automation of a Greenhouse. Mod Concep Dev Agrono. 8(4). MCDA. 000691. 2021. DOI: 10.31031/MCDA.2021.08.000691

Copyright@ Divas Karimanzira. This article is distributed under the terms of the Creative Commons Attribution 4.0 International License, which permits unrestricted use and redistribution provided that the original author and source are credited.

\section{Divas Karimanzira* and Thomas Rauschenbach}

Fraunhofer IOSB, Am Vogelherd 90, 98693 Ilmenau, Germany

\section{Opinion}

In reality, greenhouse cultivation is an adaptive process, where the grower observes his crops and adapt the environmental conditions accordingly through experience and guess work. Manual observation and control are associated with sub-optimality and delays. To achieve a fully automated greenhouse, a modified hierarchical control structure as shown in Figure 1 adopted from Van Straten et al. [1] is required.

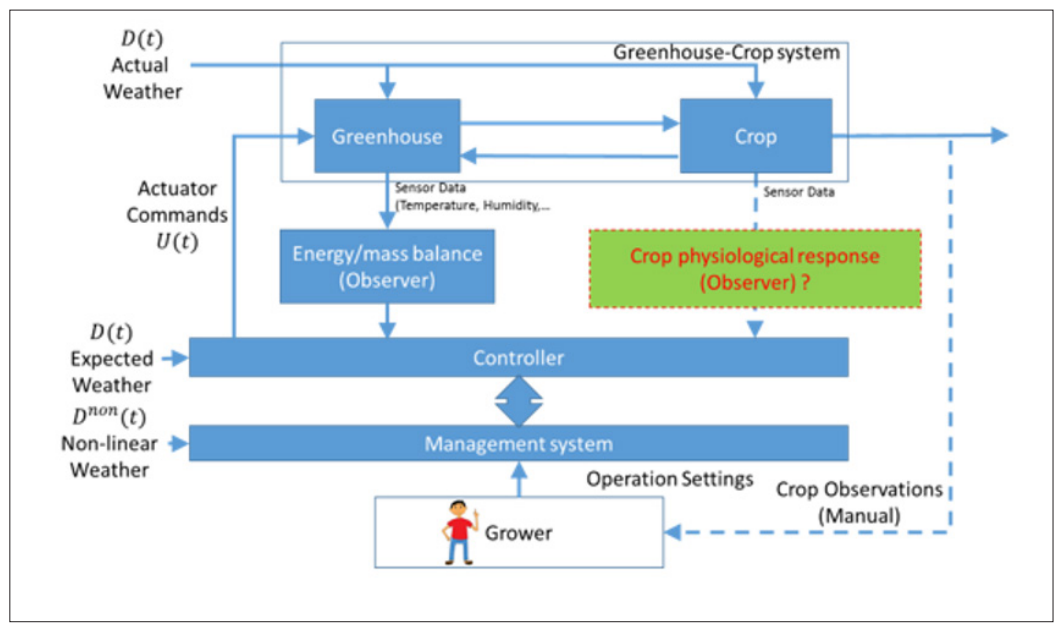

Figure 1: A modified version of the commonly used hierarchical control structure of current greenhouse-crop systems (original version Van Straten et al. [1]).

As shown in Figure 1, in the original control structure Van Straten et al. [1], crop growth state observations (or predictions) are taken manually by experienced growers, rather than sensors or computerized observers. Observers for energy/mass transportation are employed to help controller make decisions, most existing greenhouse models focus on this topic. However, data and information about the growth state of the crops (marked by a dotted line rectangle) is rarely used by the controller. Another drawback is that in most of the physical crop models that are used, typically requires a lot of parameters to be defined. The best example is the tomato growth model, TOMGRO [2]. In version 3.0, it has 574 parameters. As we know in control engineering, a vast number of parameters in the model is associated not only with high computational load, but also poor model feasibility in applications [3].

In our opinion these drawbacks as identified above can only be addressed by a new observer building strategy for greenhouse crop growth to be put in the rectangle marked by the dotted line in Figure 1. Based on the information from the growth observer, and depending on the size, type and structure of the greenhouse the controllers will manage factors such as, vents, heating, cooling, lighting, temperature, $\mathrm{CO}_{2}$, irrigation, roof to create optimal conditions for the crops in real-time. Due to mis-controls of greenhouse environment, large economic losses to growers may incur [4]. Fast and precision control responses based on the internal and structural requirements of crops are essential, and the research of crop physiological information embedded observer becomes crucial. Recently, there have been very encouraging 
studies on sensing technologies for crop physiological information. Misra et al. [5] \& Ajigboye et al. [6] have shown that chlorophyll fluorescence has become biological probes to investigate the physical status of high plants such as wheat or tomatoes.

Therefore, our expectation is that chlorophyll fluorescence and leaf gas exchanges parameters and other derived physiological parameters can be used as a powerful tool to reveal crop growth status in response to changes of greenhouse environmental conditions [7] to enable adaptive control without the grower in between.

Our proposed state observer modeling strategy for greenhouse crop growth is to take a well proven crop physiological model such as TOMGRO simulate it under optimal condition for crop growth, collect data from it to create a Long-term memory (LSTM) based reference model to be used as the observer. The data collected will include physiological parameters photosynthesis, water potential, and leaf gas exchange, and the environmental parameters such as, temperature, $\mathrm{CO}_{2}$ concentration, Vapor Pressure Deficit (VPD), lighting intensity etc., and the growth parameters will include plant height, leaf area, fresh weight, and dry weight. The physiological parameters and the environmental parameters will be used as the inputs to the model and the growth parameters as the output of the LSTM model. A LSTM is a Recurrent Neural Network (RNN) architecture that is well-suited to classify, process and predict time series given time lags of unknown duration. This makes LSTM superior over alternative RNNs, hidden Markov models and other sequence learning methods. A two-step method based on Long Short-Term Memory (LSTM) will be used to detect when the control behavior needs to be adjusted. In a first step, the LSTM is employed to learn the normal time series patterns and to predict future values of the optimally trained TOMGRO model. This is followed by the anomaly detection in the second step, which is performed by computing anomaly scores from the prediction errors. The first results of the observer are shown in Figure 2.

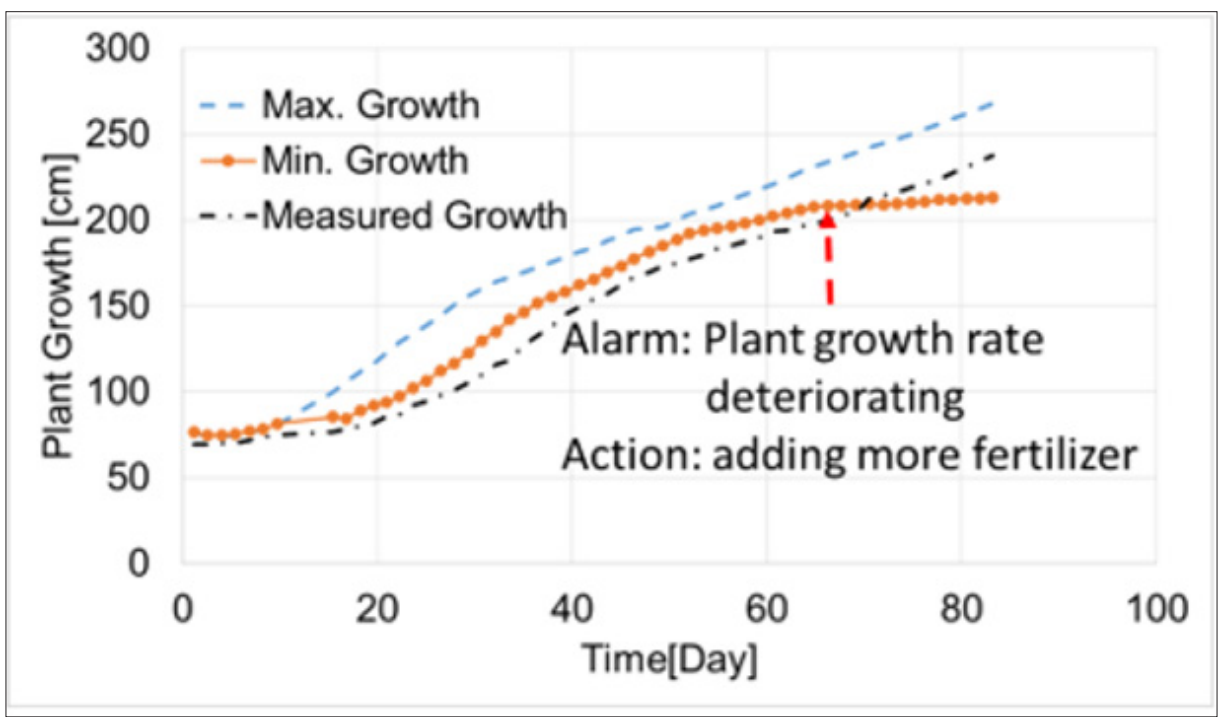

Figure 2: Results of tomato growth observation for early warning.

Benefits of greenhouse automation using observers are vast and plentiful, which include reduced the amount of work that needs to be done manually, know exactly the current situation of the greenhouse and make data driven decisions based on facts and not assumptions, and increase quality and yield through precise control, and learn from previous crop cycles.

\section{References}

1. Van Straten G, vanWilligenburg LG, Van Henten EJ, Van Ooteghem RJC (2010) Optimal control of greenhouse cultivation. Boca Raton, FL: CRC Press; Taylor \& Francis, UK.

2. Jones JW, Kenig A, Vallejos CE (1999) Reduced state-variable tomato growth model. Trans of the ASAE 42(1): 255-265.
3. Speetjens SL, Stigter JD, Van Straten G (2009) Towards an adaptive model for greenhouse control. Comput Electron Agric 67(1-2): 1-8.

4. Moriyuki S, Fukuda H (2016) High-throughput growth prediction for Lactuca sativa L. seedlings using chlorophyll fluorescence in a plant factory with artificial lighting. Front Plant Sci 7: 394.

5. Misra AN, Misra M, Singh R (2012) "Chlorophyll fluorescence in plant biology," in Biophysics, Chapter 7, Misra AN (Ed.), (Shanghai: InTech), China, pp: 171-192.

6. Ajigboye 00, Bousquet L, Murchie EH, Ray RV (2016) Chlorophyll fluorescence parameters allow the rapid detection and differentiation of plant responses in three different wheat pathosystems. Funct Plant Biol 43(4): 356-369.

7. Nishina H (2015) Development of speaking plant approach technique for intelligent greenhouse. Agric Agric Sci Proc 3: 9-13.

For possible submissions Click below: 